International Journal of Advanced Astronomy, $7(2)(2019) 25-38$
International Journal of Advanced Astronomy
SPC
Website: $w$ ww.sciencepubco.com/index.php/IJAA
Research paper

\title{
Locations of Lagrangian points and periodic orbits around triangular points in the photo gravitational elliptic restricted three-body problem with oblateness
}

\author{
Ancy Johnson ${ }^{1}$, Ram Krishan Sharma ${ }^{1 *}$ \\ ${ }^{1}$ Department of Aerospace Engineering, Karunya Institute of Technology and Sciences, Coimbatore - 641114, Tamilnadu, India \\ *Corresponding author E-mail: ramkrishansharma@gmail.com
}

\begin{abstract}
Locations of the Lagrangian points are computed and periodic orbits are studied around the triangular points in the photogravitational elliptic restricted three-body problem (ER3BP) by considering the more massive primary as the source of radiation and smaller primary as an oblate spheroid. A new mean motion taken from Sharma et al. [13] is used to study the effect of radiation pressure and oblateness of the primaries. The critical mass parameter $\mu_{c}$ that bifurcates periodic orbits from non-periodic orbits tends to reduce with radiation pressure and oblateness. The transition curves defining stable region of orbits are drawn for different values of radiation pressure and oblateness using the analytical method of Bennet [14]. Tadpole orbits with long- and short- periodic oscillations are obtained for Sun-Jupiter and SunSaturn systems.
\end{abstract}

Keywords: Critical Mass; ER3BP; Oblateness; Oblate Spheroid; Lagrangian Points; Photogravitational; Radiation Pressure; Transition Curve; Tadpole Orbits.

\section{Introduction}

ER3BP is defined as the motion of a small particle in the gravitational field of two bodies called primaries that move in elliptic orbits about their center of mass in a plane. Like circular restricted three-body problem, it does not possess Jacobi integral (Szebehely, [4]). It contains five equilibrium points, which lie in the plane of motion of the primaries. Three points $\left(\mathrm{L}_{1}, \mathrm{~L}_{2}, \mathrm{~L}_{3}\right)$ are collinear with the primaries and two points $\left(\mathrm{L}_{4}, \mathrm{~L}_{5}\right)$ form equilateral triangles with them. An application of this model can be seen in the motion of the Trojan asteroids around the triangular point $\mathrm{L}_{4}$ in the Sun-Jupiter system. The asteroids in this case are only influenced by the gravitational forces of the Sun and Jupiter, and the orbit of Jupiter around the Sun moves in an elliptic orbit.

Broucke [5] studied the effects of the stability of periodic orbits in the elliptic restricted three-body problem. By considering the more massive primary (Sun) as the source of radiation and smaller primary as an oblate spheroid, Sahoo and Ishwar [2] studied the effects of the stability of the Lagrangian points in the ER3BP. A study of equilibrium points of the ER3BP is made, when the oblate primaries of masses $m_{1}$ (larger) and $m_{2}$ are moving in elliptic orbits with eccentriciy e and mean motion $n$ with their equatorial planes coincident with the plane of motion was studied by Isravel and Sharma [8].

Raheem and Singh [7] investigated the stability of equilibrium points under the influence of small perturbations in the Coriolis and the centrifugal forces, together with the effects of oblateness and radiation pressures of the primaries. It was found that collinear points remain unstable. It is also seen that triangular points are stable for $0 \leq \mu<\mu_{\mathrm{c}}$ and unstable for $\mu_{\mathrm{c}} \leq \mu<\frac{1}{2}$, where $\mu_{\mathrm{c}}$ is the critical mass parameter and depend on the perturbations.

Subba Rao and Sharma [6] studied the planar circular restricted three-body problem by considering the more massive primary as an oblate spheroid with its equatorial plane coincident with the plane of motion. It was found that $\mu_{\text {critical }}$ is less than $\mu_{0}=0.03852 \ldots$ with oblateness of the more massive primary. Isravel and Sharma [8] studied the periodic orbits in oblate Mars and its moons system in the framework of elliptic restricted three-body problem. Transition curves which separate the stable region from the unstable region were found. Both the primaries were considered to be oblate and equilibrium points were calculated.

Bennet [14] used an analytical method to draw transition curve that can separate the stable region from the unstable region. Ruth and Sharma [10] studied periodic orbits in the elliptic restricted three-body problem by considering the radiation pressure of more massive and oblateness of smaller primary. The mean motion was derived by averaging the distance between the primaries over a revolution in terms of the mean anomaly. The tadpole orbits were generated.

In this paper, the effect of radiation pressure of the more massive primary and oblateness of the smaller primary are studied with a new mean motion. The critical mass parameter $\mu_{\mathrm{c}}$ is determined. Tadpole orbits are obtained for different Sun-Planet systems. 


\section{Equation of motion}

We consider the two bodies of larger mass $\left(\mathrm{m}_{1}\right)$ and smaller mass $\left(\mathrm{m}_{2}\right)$ rotating about their centre of mass in elliptic Keplerian orbits in a plane. The third body of infinitesimal mass is moving under their gravitational influence in the same plane. Assuming a system of canonical units, the sum of the masses of the primaries and the semi-major axis are unity (Broucke, [5]). Let the mass of the smaller primary is $\mu=$ $\frac{m_{1}}{m_{1}+m_{2}}$ and the more massive primary is $1-\mu=\frac{m_{2}}{m_{1}+m_{2}}$.

The relative distance between the primaries is given by the conic equation

$\mathrm{r}=\frac{\mathrm{p}}{1+\mathrm{ecos} \mathrm{f}}$

where $\mathrm{f}$ is the true anomaly, $\mathrm{p}$ is the semi-latus rectum a $\left(1-\mathrm{e}^{2}\right)$ with " $\mathrm{a}$ " being the semi major axis.

The planar equations of motion in the rotating-pulsating coordinate system are (Szebehely [4])

$x^{\prime \prime}-2 y^{\prime}=\frac{1}{(1+e \cos f)} \Omega_{x}, y^{\prime \prime}+2 x^{\prime}=\frac{1}{(1+e \cos f)} \Omega_{y}$,

The force function is given as (Sahoo and Ishwar [2]; Isravel and Sharma, [8])

$\Omega=\frac{1}{2}\left(x^{2}+y^{2}\right)+\frac{1}{\widetilde{n}^{2}}\left\{\frac{(1-\mu) q}{r_{1}}+\frac{\mu}{r_{2}}+\frac{\mu A_{2}}{2 r_{2}^{3}}\right\}$, with $A_{2}=\frac{R_{e}^{2}-R_{p}^{2}}{5 R^{2}}=\frac{J_{2} R_{e}^{2}}{R^{2}}$,

where $\mathrm{R}_{\mathrm{e}}$ is the equatorial radius, $\mathrm{R}_{\mathrm{p}}$ is the polar radius, $\mathrm{J}_{2}$ is the second zonal harmonic constant of the more massive primary and $\mathrm{R}$ is the distance between primaries. The positions of the primaries with respect to the third body are:

$r_{1}^{2}=(x-\mu)^{2}+y^{2}, r_{2}^{2}=(x+1-\mu)^{2}+y^{2}$,

$\mathrm{q}=1-5.6 * \frac{10^{-5}}{\mathrm{a} \delta} \mathrm{k}$

Where a is the particle radius, $\delta$ is the density, and $\mathrm{k}$ is the solar radiation pressure efficiency factor.

\subsection{Mean motion}

The mean motion (n) is the speed of the angular motion of one complete revolution of a body around another. $\mathrm{n}$ is constant (i.e. $\mathrm{n}=1)$ for CR3BP (Szebehely [4]) under suitable choice of units.

The problem of previous mean motions used was that when there is no perturbation, it depended on eccentricity which contradicts the Keplerian motion. The problem was addressed by Sharma et al. [13] as:

The new mean motion is obtained by adding the secular motion due to $\mathrm{J}_{2}$.

$\frac{d M_{S}}{d t}=n\left[1+\frac{3 J_{2}}{2 a^{2}\left(1-e^{2}\right)^{\frac{3}{2}}}\right], \frac{d \omega_{S}}{d t}=n\left[\frac{3 J_{2}}{a^{2}\left(1-e^{2}\right)^{2}}\right]$,

$\frac{\mathrm{d} \Omega_{\mathrm{S}}}{\mathrm{dt}}=\mathrm{n}\left[\frac{-3 \mathrm{~J}_{2}}{2 \mathrm{a}^{2}\left(1-\mathrm{e}^{2}\right)^{2}}\right]$.

Using the relationship, $\mathrm{n} d t=(1-\mathrm{e} \cos \mathrm{E}) \mathrm{dE}$, in Eqs. (1) and averaging over one revolution:

$\frac{1}{2 \pi} \int_{0}^{2 \pi} \mathrm{dM}_{\mathrm{S}}=\frac{1}{2 \pi} \int_{0}^{2 \pi}\left[1+\frac{3 \mathrm{~J}_{2}}{2 \mathrm{a}^{2}\left(1-\mathrm{e}^{2}\right)^{\frac{3}{2}}}\right](1-\mathrm{e} \cos \mathrm{E}) \mathrm{dE}$,

$\frac{1}{2 \pi} \int_{0}^{2 \pi} d \omega_{S}=\frac{1}{2 \pi} \int_{0}^{2 \pi}\left[\frac{3 J_{2}}{a^{2}\left(1-e^{2}\right)^{2}}\right](1-e \cos E) d E$,

$\frac{1}{2 \pi} \int_{0}^{2 \pi} d \Omega_{S}=\frac{1}{2 \pi} \int_{0}^{2 \pi}\left[\frac{-3 J_{2}}{2 a^{2}\left(1-e^{2}\right)^{2}}\right](1-e \cos E) d E$,

we get

$$
\begin{aligned}
& \bar{n}=1+\frac{3 J_{2}}{2 a^{2}\left(1-e^{2}\right)^{\frac{3}{2}}}, \\
& \Delta \omega_{S}=\frac{3 J_{2}}{a^{2}\left(1-e^{2}\right)^{2}}, \\
& \Delta \Omega_{S}=-\frac{3 J_{2}}{2 a^{2}\left(1-e^{2}\right)^{2}},
\end{aligned}
$$

Mean motion including the precession effect due to oblateness in view of (6) and (7) is calculated as:

$\tilde{n}=\bar{n}+\Delta \omega_{S}+\Delta \Omega_{S}=1+\frac{3 A_{2} R^{2}}{2 a^{2}\left[\left(1-e^{2}\right) R_{e}\right]^{2}}\left(1+\sqrt{1-e^{2}}\right)$ 
which gives $\tilde{\mathrm{n}}=1$ for unperturbed case.

\section{The collinear equilibrium points}

The influence of the effect of radiation pressure and oblateness of the primaries causing the shift in the distances of the collinear points $\mathrm{L}_{1}$, $\mathrm{L}_{2}, \mathrm{~L}_{3}$ is studied for some of the Sun and planets systems and Jupiter, Saturn and its satellite systems. Considerable difference is found when the larger primary is considered as source of radiation and smaller primary is considered as an oblate spheroid.

\subsection{Position of $L_{1}, L_{2}, L_{3}$}

The Lagrangian points $\mathrm{L}_{1}, \mathrm{~L}_{2}, \mathrm{~L}_{3}$ are obtained from $\Omega_{\xi}=0$ and $\mathrm{y}=0$ i.e.

$\xi+\frac{1}{\tilde{n}^{2}}\left\{\frac{-(1-\mu)(\xi-\mu) q}{r_{1}{ }^{3}}-\frac{\mu(\xi+1-\mu)}{r_{2}{ }^{3}}-\frac{3 \mu A_{2}(\xi+1-\mu)}{2 r_{2}^{5}}\right\}=0$

Case (i) $L_{1}$ location

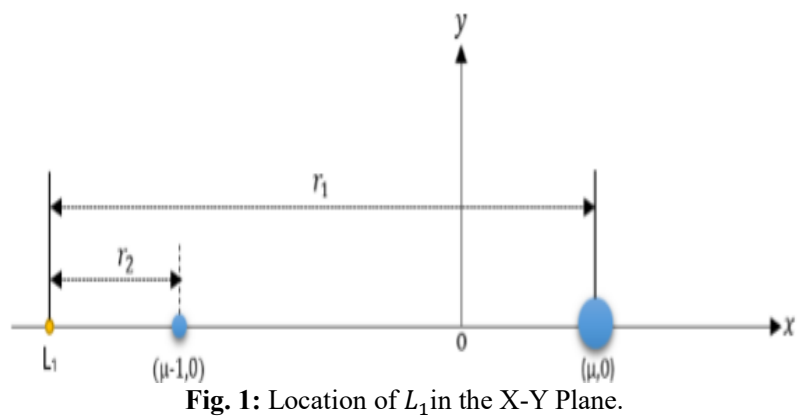

Substituting $r_{2}=\rho, r_{1}=\rho+1, \varepsilon=\mu-1-\rho$ in the above equation (9) and simplifying, we get

$-2 \tilde{n}^{2} \rho^{7}+\rho^{6}(2 \mu-6) \tilde{n}^{2}+\rho^{5}(4 \mu-6) \tilde{n}^{2}+\rho^{4}\left(\left[2 q(1-\mu)+2 \tilde{n}^{2}(\mu-1)+2 \mu\right]+4 \mu \rho^{3}+\rho^{2}\left(2+3 A_{2}\right) \mu+6 A_{2} \mu \rho+3 A_{2} \mu=0\right.$

$\rho$ can be obtained by applying Newton-Raphson's method to Eq. (10) and the abscissa of the Lagrangian point $L_{1}$ is calculated from $\varepsilon=\mu-$ $1-\rho$.

Case (ii) $L_{2}$ location

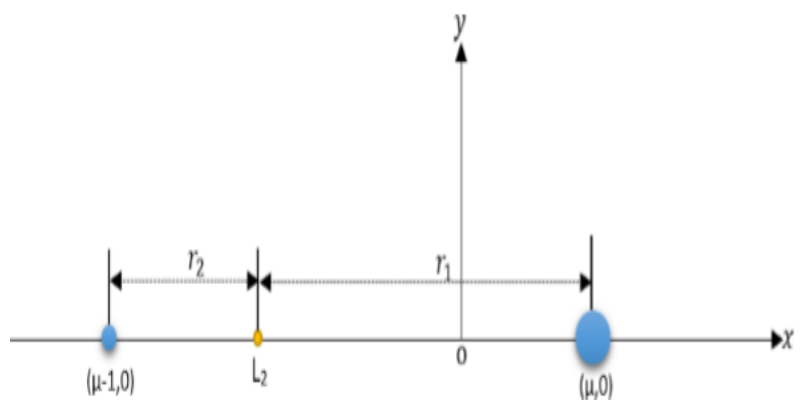

Fig. 2: Location of $L_{2}$ in the X-Y Plane.

Substituting $r_{2}=\rho, r_{1}=1-\rho, \varepsilon_{2}=\mu+\rho-1$ in the above equation (9) and simplifying, we get

$2 \tilde{n}^{2} \rho^{7}+\rho^{6}(2 \mu-6) \tilde{n}^{2}+\rho^{5}(6-4 \mu) \tilde{n}^{2}+\rho^{4}\left[2 q(1-\mu)+2 \tilde{n}^{2}(\mu-1)-2 \mu\right]+4 \mu \rho^{3}-\rho^{2}\left(2+3 A_{2}\right) \mu+6 A_{2} \mu \rho-3 A_{2} \mu=0$

$\rho$ can be obtained by applying Newton-Raphson's method to Eq. (11) and the abscissa of the Lagrangian point $L_{2}$ is calculated from $\varepsilon=\mu+\rho-1$.

Case (iii) $L_{3}$ location

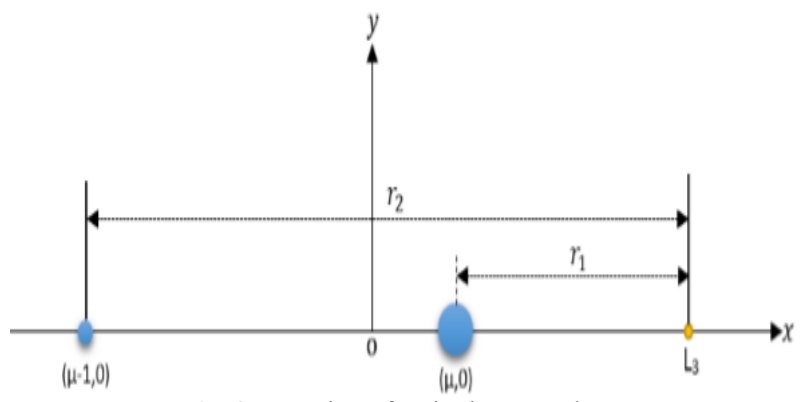

Fig. 3: Location of $L_{3}$ in the X-Y Plane. 
Substituting $r_{1}=\rho, r_{2}=1+\rho, \varepsilon=\mu+\rho$ in the above equation (9) and simplifying, we get

$2 \tilde{n}^{2} \rho^{7}+\rho^{6}(2 \mu+8) \tilde{n}^{2}+\rho^{5}(12+8 \mu) \tilde{n}^{2}+\rho^{4}\left[2 q(\mu-1)+\tilde{n}^{2}(12 \mu+8)-2 \mu\right]+\rho^{3}\left[\tilde{n}^{2}(8 \mu+2)-8 q+8 \mu q-\right.$

$4 \mu]+\rho^{2}\left(2 \tilde{n}^{2} \mu+12 q \mu-2 \mu-12 q-3 A_{2} \mu\right)+(8 q \mu-8 q) \rho+2 q(\mu-1)=0$

$\rho$ can be obtained by applying Newton-Raphson's method to Eq. (12) and the abscissa of the Lagrangian point $L_{3}$ is calculated from $\varepsilon=\mu+\rho$.

\section{Location of triangular points}

We have $\Omega_{\xi}=0, \Omega_{\eta}=0$. Therefore, the gradients of the potential are given by

$\xi-\frac{1}{\tilde{n}^{2}}\left\{\frac{(1-\mu)(\xi-\mu) q}{r_{1}{ }^{3}}+\frac{\mu(\xi+1-\mu)}{r_{2}{ }^{3}}+\frac{3 \mu A_{2}(\xi+1-\mu)}{2 r_{2}^{5}}\right\}=0$

$\eta\left(1-\frac{1}{\tilde{n}^{2}}\left\{\frac{(1-\mu) q}{r_{1}{ }^{3}}+\frac{\mu}{r_{2}{ }^{3}}+\frac{3 \mu A_{2}}{2 r_{2}^{5}}\right\}\right)=0$

By Solving, we arrive at the following equation

$r_{1}^{3}=\frac{q}{\tilde{n}^{2}}$

Substituting (15) in (14), we get

$r_{2}^{3}=\frac{1}{\tilde{n}^{2}}\left(1+\frac{3 A 2}{2 r_{2}^{2}}\right)$.

Substituting $\mathrm{K}_{2}=3 \mathrm{R}^{2} /\left(\mathrm{a}^{2} \mathrm{Re}_{\mathrm{e}}^{2}\right)$ in (8) and simplifying the mean motion expression, we have

$\tilde{n}^{2}=1+2 K_{2} A_{2}\left[1+\frac{7}{4} e^{2}\right]$.

Solving (16)

$2 r_{2}{ }^{5} \tilde{n}^{2}-2 r_{2}{ }^{2}-3 A_{2}=0$.

Let $r_{2}^{2}=1+\eta$.

Substituting $r_{2}$ in (18) and solving for $\eta$. We get

$\eta=\frac{2+3 A_{2}-2 \tilde{n}^{2}}{5 \tilde{n}^{2}-2}$.

Substituting (17), we get

$\eta=A_{2}\left[1-\frac{4}{3} K_{2}\left(1+\frac{7}{4} e^{2}\right)\right]$.

As $r_{2}^{2}=1+\eta$, it becomes

$r_{2}{ }^{2}=1+A_{2}\left[1-\frac{4}{3} K_{2}\left(1+\frac{7}{4} e^{2}\right)\right]$

For $r_{1}^{2}=1-\xi, \mathrm{q}=1-\varepsilon$ and using (15) and (17), we get

$r_{1}^{2}=1+\frac{2}{3} \varepsilon-\frac{4}{3} K_{2} A_{2}\left(1+\frac{7}{4} e^{2}\right)\left(1+\frac{2}{3} \varepsilon\right)$.

Solving and simplifying, we obtain

$\xi=\mu-\frac{1}{2}+\frac{A_{2}}{2}-\frac{\varepsilon}{3}+\frac{4}{9} A_{2} K_{2} \varepsilon$,

$\eta= \pm \frac{\sqrt{3}}{2}\left[1+\frac{2}{9} \varepsilon+\frac{A_{2}}{3}-\frac{8}{9} A_{2} K_{2}-\frac{8}{27} A_{2} K_{2} \varepsilon\right]$.

If the oblateness and radiation pressure terms are eliminated i.e. $A_{1}=0, \varepsilon=0$ and eccentricity e $=0$, then the system reduces to $\mathrm{CR} 3 \mathrm{BP}$ and the location of the triangular equilibrium points are:

$\varepsilon=\mu-\frac{1}{2}$ and $\eta= \pm \frac{\sqrt{3}}{2}$ 


\section{Stability of triangular points}

Let $(\xi, \eta)$ be the position of one of the equilibrium point. After a small displacement, the coordinates of the small particle are $(\xi+u, \eta+$ $v)$, where $u, v$ are small. The variational equations of motion are:

$u^{\prime \prime}-2 v^{\prime}=\frac{1}{1+e \cos f}\left[u \Omega_{\xi \xi}+v \Omega_{\xi \eta}\right]$

$v^{\prime \prime}+2 u^{\prime}=\frac{1}{1+e \cos f}\left[v \Omega_{\eta \eta}+u \Omega_{\xi \eta}\right]$

As Grebenikov [9] suggested, the variations posed by true anomaly ' $f$ ' can be eliminated by using the method of averaging on the coefficients of equations over the time period ' $2 \pi$ '.

$\frac{1}{2 \pi} \int_{0}^{2 \pi} \frac{1}{1+e \cos f} d f=\frac{1}{\sqrt{1-e^{2}}}$

Therefore (25) becomes

$u^{\prime \prime}-2 v^{\prime}=\frac{1}{\sqrt{1-e^{2}}}\left[u \Omega_{\xi \xi}+v \Omega_{\xi \eta}\right]$

$v^{\prime \prime}+2 u^{\prime}=\frac{1}{\sqrt{1-e^{2}}}\left[v \Omega_{\eta \eta}+u \Omega_{\xi \eta}\right]$

The characteristic equation is given by

$\lambda^{4}+\left[4-\frac{\left(\Omega_{x x}+\Omega_{y y}\right)}{\sqrt{1-e^{2}}}\right] \lambda^{2}+\frac{\Omega_{x x} \Omega_{y y}-\Omega_{x y}{ }^{2}}{\left(1-e^{2}\right)}=0$.

Eliminating the higher order terms and restricting to $e^{2}, A_{2}$ and $\varepsilon$ terms, we get

$\lambda^{4}+\left[4-\frac{3}{\sqrt{1-e^{2}}}+A 2\left(\frac{-8 K 2-7 e^{2} K 2}{\sqrt{1-e^{2}}}+\frac{\left(-54+36 K 2+63 e^{2} K 2\right) \mu}{18 \sqrt{1-e^{2}}}+\left(\frac{-12-44 K 2-63 e^{2} 2}{18 \sqrt{1-e^{2}}}+\frac{\left(60 K 2+63 e^{2} K 2\right) \mu}{18 \sqrt{1-e^{2}}}\right) \varepsilon\right)\right] \lambda^{2}-\frac{27 \mu}{4\left(-1+e^{2}\right)}+\frac{27 \mu^{2}}{4\left(-1+e^{2}\right)}+$

$\left(-\frac{3 \mu}{2\left(-1+e^{2}\right)}+\frac{3 \mu^{2}}{2\left(-1+e^{2}\right)}\right) \varepsilon+A 2\left(\frac{\left(-324-2052 K 2-2079 e^{2} K 2\right) \mu}{72\left(-1+e^{2}\right)}+\frac{\left(324+2160 K 2+2268 e^{2} K 2\right) \mu^{2}}{72\left(-1+e^{2}\right)}+\left(\frac{-144 K 2+252 e^{2} K 2}{72\left(-1+e^{2}\right)}+\frac{\left(-144-348 K 2-1827 e^{2} K 2\right) \mu}{72\left(-1+e^{2}\right)}+\right.\right.$

$\left.\left.\frac{\left(144+636 K 2+1827 e^{2} K 2\right) \mu^{2}}{72\left(-1+e^{2}\right)}\right) \varepsilon\right)=0$

The stability theorem (E.A.Grebenikov, 1964) states that if the condition $\sqrt{7} / 4 \leq e<1$ is satisfied by e of the orbits of the attracting masses, then the solution (29) is unstable. Hence the proof is provided by taking $\varepsilon=A_{2}=0$, imparting this in Eq. (29), the roots will be

$\lambda= \pm \sqrt{\frac{-1-e^{2}}{4} \pm \sqrt{\frac{\left(1+e^{2}\right)^{2}-27 \mu(1-\mu)}{4}}}$

Let us take the inequality

$\frac{-1-e^{2}}{4} \geq 0$

The eccentricity does not satisfy the above inequality hence it does not hold when $\sqrt{7} / 4 \leq e<1$, so Eq. (29) have either real roots or complex roots. In both the cases there must definitely be the negative real parts of roots. Then this yields that the Lagrangian solutions in Eq. (29) are stable.

Let us consider $\lambda^{2}=\Lambda$ in (29), then

$\Lambda^{2}+\left[4-\frac{3}{\sqrt{1-e^{2}}}+A 2\left(\frac{-8 K 2-7 e^{2} K 2}{\sqrt{1-e^{2}}}+\frac{\left(-54+36 K 2+63 e^{2} K 2\right) \mu}{18 \sqrt{1-e^{2}}}+\left(\frac{-12-44 K 2-63 e^{2} K 2}{18 \sqrt{1-e^{2}}}+\frac{\left(60 K 2+63 e^{2} K 2\right) \mu}{18 \sqrt{1-e^{2}}}\right) \varepsilon\right)\right] \Lambda-\frac{27 \mu}{4\left(-1+e^{2}\right)}+\frac{27 \mu^{2}}{4\left(-1+e^{2}\right)}+$ $\left(-\frac{3 \mu}{2\left(-1+e^{2}\right)}+\frac{3 \mu^{2}}{2\left(-1+e^{2}\right)}\right) \varepsilon+A 2\left(\frac{\left(-324-2052 K 2-2079 e^{2} K 2\right) \mu}{72\left(-1+e^{2}\right)}+\frac{\left(324+2160 K 2+2268 e^{2} K 2\right) \mu^{2}}{72\left(-1+e^{2}\right)}+\left(\frac{-144 K 2+252 e^{2} K 2}{72\left(-1+e^{2}\right)}+\frac{\left(-144-348 K 2-1827 e^{2} K 2\right) \mu}{72\left(-1+e^{2}\right)}+\right.\right.$ $\left.\left.\frac{\left(144+636 K 2+1827 e^{2} K 2\right) \mu^{2}}{72\left(-1+e^{2}\right)}\right) \varepsilon\right)=0$

The critical mass parameter

$\mu_{\mathrm{c}}=\frac{1}{54}\left(27-3 \sqrt{3} \sqrt{-73+64 e^{2}+96 \sqrt{1-e^{2}}}\right)-\frac{\varepsilon}{243}(27-3 \sqrt{69})-\frac{A_{2}}{9 \sqrt{69}}(27-3 \sqrt{69})\left(69+460 K_{2}\right)$

When $\varepsilon=A_{2}=0$, Critical mass parameter obtained matches with that of Grebenikov [9] and Danby [1], i.e.

$\mu_{\mathrm{c}}=\frac{1}{54}\left(27-3 \sqrt{3} \sqrt{-73+64 e^{2}+96 \sqrt{1-e^{2}}}\right)$.

The critical mass parameter for the classical case $(\mathrm{e}=0)$ becomes 
$\mu_{\mathrm{c}}=\frac{1}{54}(27-3 \sqrt{69})=0.03852 \ldots$

The classical elliptical case has the critical mass parameter as $\mu_{c}=0.03852 \ldots$.

It may be noted from Eq. (31) that in our case the critical mass parameter decreases with the increase in oblateness and radiation pressure.

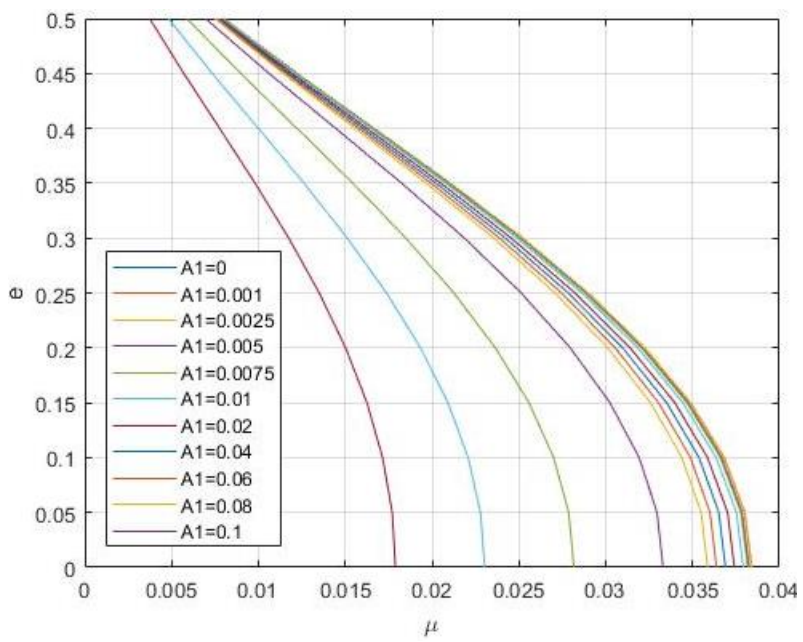

Fig. 4: Critical Mass vs. Eccentricity.

In figure (4) different curves are plotted taking eccentricity e from 0 to 0.5 vs. $\mu$ from 0 to 0.05 using Eq. (31) with the oblateness of the smaller primary as $\mathrm{A}_{2}=0, \mathrm{~A}_{2}=0.001, \mathrm{~A}_{2}=0.0025$ up to $\mathrm{A}_{2}=0.1$. The blue curve indicates classical ER3BP, where the singularity is at $\mu_{c}=$ $0.0385 \ldots$... This is one of the singularity drawn by Danby [1]. From Fig. (4), it is seen that singularities move towards left, when oblateness and radiation pressure increases. Indeed, it affects the stability of the triangular points. It is observed that when bodies are oblate and radiating, the stability decreases.

\section{Transition curve}

Using the analytical method of the Bennet [14], we get the variational equations in the vector form as

$X=\left\{\begin{array}{c}u \\ v \\ u^{\prime} \\ v^{\prime}\end{array}\right\} X^{\prime}=\left\{\begin{array}{c}u^{\prime} \\ u^{\prime \prime} \\ v^{\prime} \\ v^{\prime \prime}\end{array}\right\} p(f, e)=\left(\begin{array}{cccc}0 & 0 & 1 & 0 \\ 0 & 0 & 0 & 1 \\ \varphi \Omega_{\varepsilon \varepsilon} & \varphi \Omega_{\varepsilon \eta} & 0 & 2 \\ \varphi \Omega_{\eta \varepsilon} & \varphi \Omega_{\eta \eta} & -2 & 0\end{array}\right)$

where $\varphi=\frac{1}{1+e \cos f}$.

In matrix notation

$X^{\prime}=p X$

Utilizing the Floquet theory with the periodic coefficients, the form of the solution is

$$
X_{k}=y_{k} e^{\lambda_{k} f}
$$

where $y_{k}$ is periodic with period T. Discarding the subscript and differentiating Eq. (34) with respect to f and substituting Eq. (33) into it, we get

$y^{\prime}=(p-I \lambda) y$

Expanding $\mathrm{y}, \lambda$ and the matrix $p$ into

$y=y^{(0)}+y^{(1)} e+y^{(2)} e^{2}+\cdots$

$\lambda=\lambda_{0}+\lambda_{1} e+\lambda_{2} e^{2}+\cdots$

$p(f, e)=p^{(0)}+p^{(1)} e+p^{(2)} e^{2}+\cdots$

where

$p^{(m)}=(-\cos f)^{(m)} \mathrm{cm}=1,2,3$. 
$p^{(0)}=\left(\begin{array}{cccc}0 & 0 & 1 & 0 \\ 0 & 0 & 0 & 1 \\ \Omega_{\varepsilon \varepsilon} & \Omega_{\varepsilon \eta} & 0 & 2 \\ \Omega_{\eta \varepsilon} & \Omega_{\eta \eta} & -2 & 0\end{array}\right) c=\left(\begin{array}{cccc}0 & 0 & 0 & 0 \\ 0 & 0 & 0 & 0 \\ \Omega_{\varepsilon \varepsilon} & \Omega_{\varepsilon \eta} & 0 & 0 \\ \Omega_{\eta \varepsilon} & \Omega_{\eta \eta} & 0 & 0\end{array}\right)$.

Now substituting Eq. (36) into (34), we get

$y^{\prime(0)}+y^{\prime(1)} e+y^{\prime(2)} e^{2}+\cdots=\left\{\left[\left(p^{(0)}+p^{(1)} e+p^{(2)} e^{2}+\cdots\right)-I\left(\lambda_{0}+\lambda_{1} e+\lambda_{2} e^{2}+\cdots\right)\right]\left(y^{(0)}+y^{(1)} e+y^{(2)} e^{2}+\cdots\right)\right\}$

The particular solution is

$y^{(n)}=\sum_{k=-n}^{+n}\left(\begin{array}{l}a_{1}^{(n, k)} \\ a_{2}^{(n, k)} \\ a_{3}^{(n, k)} \\ a_{4}^{(n, k)}\end{array}\right) e^{i k f} n=0,1,2, \ldots$

If the zeroth order solution is assumed to have a constant vector, then the non-homogenous terms have the frequencies $\leq n / 2 \pi$ for the $\mathrm{n}^{\text {th }}$ order equation.

Equating Eq. (38) according to the coefficient of e and its powers and substituting Eq. (39) in it, we get

$\left(I \lambda_{0}-p^{(0)}\right) a^{(0,0)}=0$

$\left(I \lambda_{0}-p^{(0)}\right) a^{(1,0)}=-\lambda_{1} a^{(0,0)}$

$\left(I\left(\lambda_{0}+i\right)-p^{(0)}\right) a^{(1,+1)}=-\frac{1}{2} c a^{(0,0)}$

$\left(I\left(\lambda_{0}-i\right)-p^{(0)}\right) a^{(1,-1)}=-\frac{1}{2} c a^{(0,0)}$

$\left(I \lambda_{0}-p^{(0)}\right) a^{(2,0)}=-\lambda_{1} a^{(1,0)}+\left(\frac{1}{2} c-I \lambda_{2}\right) a^{(0,0)}-\frac{1}{2} c\left(a^{(1,+1)}+a^{(1,-1)}\right)$

Since the solution for the elements of $a^{(0,0)} \neq 0$, we have from the first of Eqs. (40)

$\operatorname{det}\left(I \lambda_{0}-p^{(0)}\right)=0$

From Eq. (41), we get the characteristic Equation

$\lambda^{4}+\left(4-\Omega_{\varepsilon \varepsilon}-\Omega_{\eta \eta}\right) \lambda^{2}+\left(\Omega_{\varepsilon \varepsilon} \Omega_{\eta \eta}-\Omega_{\varepsilon \eta}^{2}\right)=0$.

The relation (42) can be written for the classical CR3BP as

$\lambda_{0}^{4}+\lambda_{0}^{2}+\frac{27}{4} \mu(1-\mu)=0$.

Now taking the determinant of the coefficients on the left of the second of Eq. (40) to be zero by the non-homogenous terms on the right replacing any column of it, then

$\operatorname{det}\left(I \lambda_{0}-p^{(0)}\right)+\lambda_{1} a^{(0,0)}=0$.

Since the determinant cannot be zero, we conclude $\lambda_{1}=0$.

From the third and fourth Eqs. of (40), the values of $a^{(1,+1)}$ and $a^{(1,-1)}$ can be calibrated and it is substituted in the last Eq. of (40) to yield

$\left(I \lambda_{0}-p^{(0)}\right) a^{(2,0)}=\frac{1}{4} c\left\{\left[I\left(\lambda_{0}+i\right)-p^{(0)}\right]^{-1}+\left[I\left(\lambda_{0}-i\right)-p^{(0)}\right]^{-1}\right\} c a^{(0,0)}+\left(\frac{1}{2} c-I \lambda_{2}\right) a^{(0,0)}$

In the above equation, considering the real part of any one of the complex conjugate terms on the first part of equation on the left, we attain

$\left(I \lambda_{0}-p^{(0)}\right) a^{(2,0)}=\left\{\frac{1}{2} c R_{e}\left[I\left(\lambda_{0}-i\right)-p^{(0)}\right]^{-1} c a^{(0,0)}+\left(\frac{1}{2} c-I \lambda_{2}\right)\right\} a^{(0,0)}$

When this relation is mathematically executed, it is of the form:

$\lambda_{2}=-\left[\frac{\left(Q^{2}-4 R-16\right) \lambda_{0}^{2}+A_{0} F_{0}+A_{1} F_{1}+A_{2} F_{2}}{4\left(Q^{2}-4 Q-4 R\right) \lambda_{0}^{2}+32 R} \lambda_{0}\right]$

The solution of the system is

$\lambda=\lambda_{0}+\lambda_{2} e^{2}$, 
where

$A_{0}=\left[(Q+4)^{2}(Q-4)-4 Q R\right] \lambda_{0}^{2}-R(Q+4)-4 R^{2}$

$A_{1}=-8 \lambda_{0} R\left[2 \lambda_{0}^{2}-(Q+4)\right]$,

$A_{2}=-\lambda_{0}^{2} R\left[Q^{2}-4 R-16\right]$,

$N=\lambda_{0}^{2}\left[4 Q^{2}+8 Q+4-16 R\right]-4 R+(Q+1)^{2}$,

$F_{0}=1 / N\left[\lambda_{0}^{2}(Q+1)+(Q+1)+2 R\right]$

$F_{1}=-\lambda_{0} / N\left[2 \lambda_{0}^{2}+(Q+3)\right.$,

$F_{2}=-\frac{1}{N\left[6 \lambda_{0}^{2}-(Q+1)\right]}$,

And

$Q=-\left(4-\Omega_{\varepsilon \varepsilon}-\Omega_{\eta \eta}\right)$

$R=\Omega_{\varepsilon \varepsilon} \Omega_{\eta \eta}-\Omega_{\varepsilon \eta}^{2}$

The transition curve is rendered by equating the value of periodic solutions to the expression of the characteristic exponents. In the range of interest $0 \leq \mu \leq 1$, the periodic solution produces

$\lambda^{*}= \pm i / 2$

Substitute above in Eq. (45) by making $\lambda^{*}=\lambda$, Eq. (45) becomes

$\pm \frac{i}{2}=\lambda_{0}+\lambda_{2} e^{2}$

Squaring on both the sides, we get

$-1 / 4=\left(\lambda_{0}+\lambda_{2} e^{2}\right)^{2}$

Eq. (46) generates the transition curve which separates the stable region from the unstable region as shown in 5 to 8 , where the spacecraft will be stable in the vicinity of the region inside the curve until the singularity $\mu=0.0385 .$. , while the area outside the curve is unstable. The transition curve obtained deviates from the numerical results as the eccentricity increases and the oblateness of the primaries affects the stability of the triangular points. When the oblateness increases, the stability decreases.
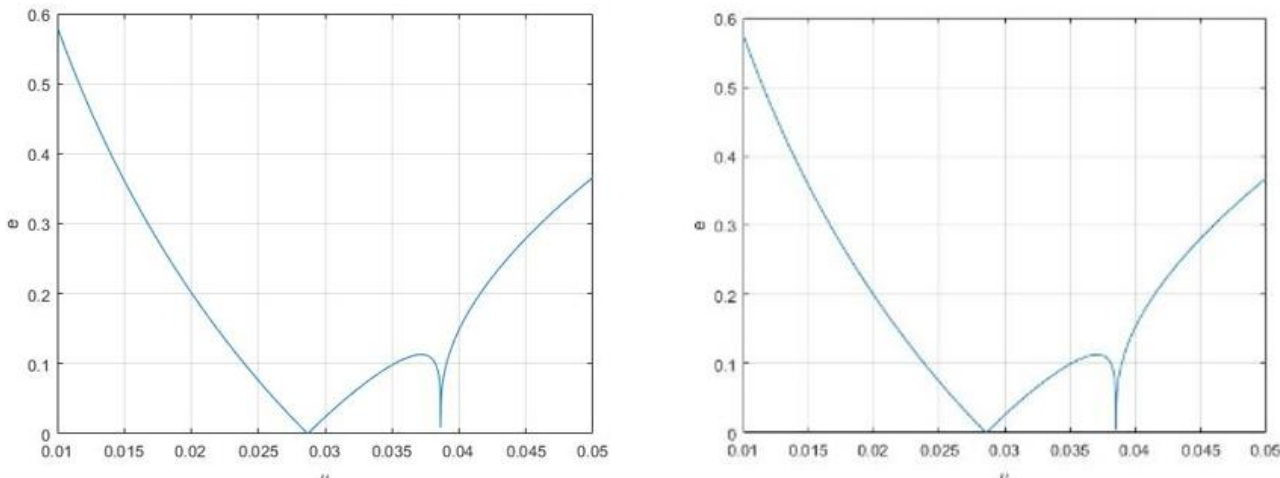

Fig. 5: Transition Curve When $A_{2}=0.003$ and $\xi=0.001$ Fig 6 Transition Curve when $A_{2}=0$ and $\xi=0$.
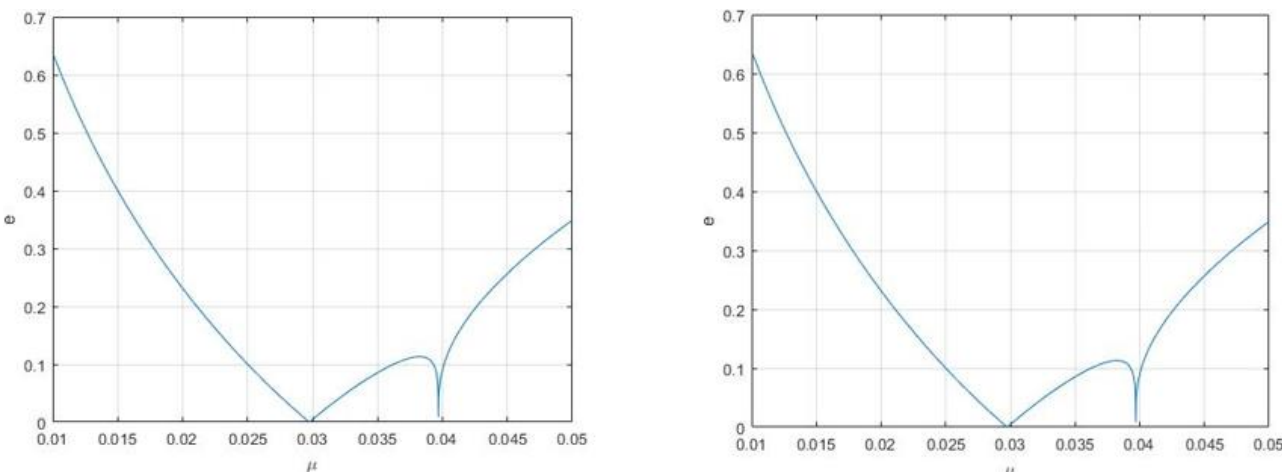

Fig. 7: Transition Curve when $A_{2}=0$ and $\Xi=0.01$ Fig 8 Transition Curve when $A_{2}=0.001$ and $\Xi=0$ 


\section{Tadpole orbits}

The motion around the triangular libration points are characterized by stable oscillations known as tadpole orbits. They constitute two kinds of orbits: a short-period epicyclical oscillation which is associated with the orbital period of the second primary and a long-period librational motion around an equilibrium point.Tadpole orbits are representatives of resonant coorbital motion that exist in the vicinity of triangular Lagrange points. In this paper, the study focuses on periodic solutions in the vicinity of these triangular libration points specifically, tadpole orbits for Sun-Jupiter and Sun-Saturn systems under the effect of oblateness of Jupiter/Saturn and radiation pressure of Sun. Tadpole orbits are constructed by solving the variational equations of motion using an analytical method.

\subsection{Periodic orbit around libration points}

For the triangular points, the gradients of the potential function are

$$
\begin{aligned}
& \Omega_{\xi \xi}=\frac{3}{4}-\frac{3 A_{2}}{2}+A_{2} K_{2}+3 A_{2} \mu-\frac{\mu}{2} \\
& \Omega_{\eta \eta}=\frac{9}{4}+\frac{3 A_{2}}{2}+7 A_{2} K_{2}+\frac{\mu}{2} \\
& \Omega_{\xi \eta}=\frac{1}{\sqrt{3}}\left[-\frac{9}{4}+\frac{3 A_{2}}{2}-5 A_{2} K_{2}+\frac{9 \mu}{2}+\frac{3 A_{2} \mu}{2}+\frac{\mu}{2}+\frac{\mu \varepsilon}{2}\right] .
\end{aligned}
$$

Eq. (47) is substituted in Eq. (28) to get

$\lambda^{4}+\left(4-\frac{3}{\sqrt{\left(1-e^{2}\right)}}-\frac{8 A_{2} K_{2}}{\sqrt{\left(1-e^{2}\right)}}-\frac{3 A_{2} \mu}{\sqrt{\left(1-e^{2}\right)}}-\frac{2 A_{2} \varepsilon}{3 \sqrt{\left(1-e^{2}\right)}}\right) \lambda^{2}+\frac{1}{\left(1-e^{2}\right)} \frac{27}{4} \mu(1-\mu)+\frac{3 \mu \varepsilon}{2\left(1-e^{2}\right)}(1-\mu)+\frac{A_{2} \mu}{72\left(1-e^{2}\right)}\left(324+2052 K_{2}+\right.$ $\left.2079 e^{2} K_{2}\right)=0$.

The eigenvalues of the above equation can be obtained as

$$
\begin{aligned}
& \lambda^{2}=-\frac{1}{2}\left(4-\frac{3}{\sqrt{\left(1-e^{2}\right)}}-\frac{8 A_{2} K_{2}}{\sqrt{\left(1-e^{2}\right)}}-\frac{3 A_{2} \mu}{\sqrt{\left(1-e^{2}\right)}}-\frac{2 A_{2} \varepsilon}{3 \sqrt{\left(1-e^{2}\right)}}\right) \pm \frac{1}{2}\left(\left(4-\frac{3}{\sqrt{\left(1-e^{2}\right)}}-\frac{8 A_{2} K_{2}}{\sqrt{\left(1-e^{2}\right)}}-\frac{3 A_{2} \mu}{\sqrt{\left(1-e^{2}\right)}}-\frac{2 A_{2} \varepsilon}{3 \sqrt{\left(1-e^{2}\right)}}\right)^{2}-\left(\frac{27}{\left(1-e^{2}\right)} \mu(1-\mu)+\right.\right. \\
& \left.\left.\frac{6 \mu \varepsilon}{\left(1-e^{2}\right)}(1-\mu)+\frac{A_{2} \mu}{18\left(1-e^{2}\right)}\left(324+2052 K_{2}+2079 e^{2} K_{2}\right)\right)\right)^{0.5}<0 .
\end{aligned}
$$

Since all the roots are imaginary, it becomes $\lambda_{1,2}= \pm i v_{1}$ and $\lambda_{3,4}= \pm i v_{2}$, then the generic solution is written as (Vanderlecht [12])

$$
\begin{aligned}
& x(f)=\alpha_{1} \cos \left(v_{1} f\right)+\alpha_{2} \sin \left(v_{1} f\right)+\alpha_{3} \cos \left(v_{2} f\right)+\alpha_{4} \sin \left(v_{2} f\right), \\
& y(f)=\beta_{1} \cos \left(v_{1} f\right)+\beta_{2} \sin \left(v_{1} f\right)+\beta_{3} \cos \left(v_{2} f\right)+\beta_{4} \sin \left(v_{2} f\right) .
\end{aligned}
$$

By substituting the above values in the general equation (49) and comparing the coefficients of the same Fourier components, we get

$$
\begin{aligned}
& \alpha_{1}=-\frac{2 \beta_{2} v_{1}+\Omega_{\xi \eta}(a, b) \beta_{1}}{v_{1}{ }^{2}+\Omega_{\xi \xi}(a, b)} \\
& \alpha_{2}=\frac{2 \beta_{1} v_{1}-\Omega_{\xi \eta}(a, b) \beta_{2}}{v_{1}{ }^{2}+\Omega_{\xi \xi}(a, b)} \\
& \alpha_{3}=\frac{-2 \beta_{4} v_{2}-\Omega_{\xi \eta}(a, b) \beta_{3}}{v_{2}{ }^{2}+\Omega_{\xi \xi}(a, b)} \\
& \alpha_{4}=\frac{2 \beta_{3} v_{2}-\Omega_{\xi \eta}(a, b) \beta_{4}}{v_{2}{ }^{2}+\Omega_{\xi \xi}(a, b)} \\
& \beta_{1}=\frac{2 \alpha_{2} v_{1}-\Omega_{\xi \eta}(a, b) \alpha_{1}}{v_{1}{ }^{2}+\Omega_{\eta \eta}(a, b)} \\
& \beta_{2}=-\frac{2 \alpha_{1} v_{1}+\Omega_{\xi \eta}(a, b) \alpha_{2}}{v_{1}{ }^{2}+\Omega_{\eta \eta}(a, b)} \\
& \beta_{3}=\frac{2 \alpha_{4} v_{2}-\Omega_{\xi \eta}(a, b) \alpha_{3}}{v_{2}{ }^{2}+\Omega_{\eta \eta}(a, b)} \\
& \beta_{4}=-\frac{2 \alpha_{3} v_{2}+\Omega_{\xi \eta}(a, b) \alpha_{4}}{v_{2}{ }^{2}+\Omega_{\eta \eta}(a, b)}
\end{aligned}
$$

The initial conditions are given as

$$
\begin{aligned}
& x_{0}=x(0)=\alpha_{1}+\alpha_{3}, x_{0}^{\prime}=x^{\prime}(0)=\alpha_{4} v_{2}+\alpha_{2} v_{1}, x_{0}^{\prime \prime}=\alpha_{3} v_{2}{ }^{2}-\alpha_{1} v_{1}{ }^{2}, \\
& y_{0}=y(0)=\beta_{1}+\beta_{3}, y_{0}^{\prime}=y^{\prime}(0)=\beta_{4} v_{2}+\beta_{2} v_{1}, y_{0}^{\prime \prime}=\beta_{3} v_{2}{ }^{2}-\beta_{1} v_{1}{ }^{2} .
\end{aligned}
$$


Substituting Eqn. (50) in these equations, we get

$x_{0}=-\frac{2 \beta_{2} v_{1}+\Omega_{\xi y}(a, b) \beta_{1}}{v_{1}{ }^{2}+\Omega_{\xi \xi}(a, b)}+\frac{-2 \beta_{4} v_{2}-\Omega_{\xi y}(a, b) \beta_{3}}{v_{2}{ }^{2}+\Omega_{\xi \xi}(a, b)}, x_{0}^{\prime}=\frac{v_{2}\left(2 \beta_{3} v_{2}-\Omega_{\xi y}(a, b) \beta_{4}\right)}{v_{2}{ }^{2}+\Omega_{\xi \xi}(a, b)}+\frac{v_{1}\left(2 \beta_{1} v_{1}-\Omega_{\xi y}(a, b) \beta_{2}\right)}{v_{1}{ }^{2}+\Omega_{\xi \xi}(a, b)}$.

And

$y_{0}=\frac{2 \alpha_{2} v_{1}-\Omega_{\xi \eta}(a, b) \alpha_{1}}{v_{1}^{2}+\Omega_{\eta \eta}(a, b)}+\frac{2 \alpha_{4} v_{2}-\Omega_{\xi \eta}(a, b) \alpha_{3}}{v_{2}{ }^{2}+\Omega_{\eta \eta}(a, b)}, y_{0}^{\prime}=-\frac{v_{2}\left(2 \alpha_{3} v_{2}+\Omega_{\xi \eta}(a, b) \alpha_{4}\right)}{v_{2}{ }^{2}+\Omega_{\eta \eta}(a, b)}-\frac{v_{1}\left(2 \alpha_{1} v_{1}+\Omega_{\xi \eta}(a, b) \alpha_{2}\right)}{v_{1}{ }^{2}+\Omega_{\eta \eta}(a, b)}$.

Solving the system of variational equations with the above equations to get the amplitudes $\alpha_{1}, \alpha_{2}, \alpha_{3}, \alpha_{4}$ and $\beta_{1}, \beta_{2}, \beta_{3}, \beta_{4}$, respectively. Let the initial conditions for the closer motion around $\mathrm{L}_{4}$ is given by $x_{0}=y_{0}=10^{-5}, x_{0}^{\prime}=y_{0}^{\prime}=10^{-5}$, then

$$
\begin{aligned}
& \beta_{1}=-\frac{\left(\Omega_{\xi \eta}-2\right) v_{1}^{2}+\Omega_{\xi \xi} \Omega_{\xi \eta}-2 \Omega_{\xi \xi}}{400000 v_{1}^{2}+100000 \Omega_{\xi \eta}^{2}} \\
& \beta_{2}=-\frac{2 v_{1}^{4}+\left(\Omega_{\xi \eta}+2 \Omega_{\xi \xi}\right) v_{1}^{2}+\Omega_{\xi \xi} \Omega_{\xi \eta}}{400000 v_{1}^{3}+100000 \Omega_{\xi \eta}^{2} \nu_{1}} \\
& \beta_{3}=-\frac{\left(\Omega_{\xi \eta}-2\right) v_{2}^{2}+\Omega_{\xi \xi} \Omega_{\xi \eta}-2 \Omega_{\xi \xi}}{400000 v_{2}^{2}+100000 \Omega_{\xi \eta}^{2}} \\
& \beta_{4}=-\frac{2 v_{2}^{4}+\left(\Omega_{\xi \eta}+2 \Omega_{\xi \xi}\right) v_{2}^{2}+\Omega_{\xi \xi} \Omega_{\xi \eta}}{400000 v_{2}^{3}+100000 \Omega_{\xi \eta}^{2} v_{2}} \\
& \alpha_{1}=-\frac{\left(\Omega_{\xi \eta}+2\right) v_{1}^{2}+\Omega_{\eta \eta} \Omega_{\xi \eta}+2 \Omega_{\eta \eta}}{400000 v_{1}^{2}+100000 \Omega_{\xi \eta}^{2}} \\
& \alpha_{2}=\frac{2 v_{1}^{4}+\left(2 \Omega_{\eta \eta}-\Omega_{\xi \eta}\right) v_{1}^{2}-\Omega_{\xi \xi} \Omega_{\xi \eta}}{400000 v_{1}^{3}+100000 \Omega_{\xi \eta}^{2} v_{1}} \\
& \alpha_{3}=-\frac{\left(\Omega_{\xi \eta}+2\right) v_{2}^{2}+\Omega_{\eta \eta} \Omega_{\xi \eta}+2 \Omega_{\eta \eta}}{400000 v_{2}^{2}+100000 \Omega_{\xi \eta}^{2}} \\
& \alpha_{4}=\frac{2 v_{2}^{4}+\left(2 \Omega_{\eta \eta}-\Omega_{\xi \eta}\right) v_{2}^{2}-\Omega_{\xi \xi} \Omega_{\xi \eta}}{400000 v_{2}^{3}+100000 \Omega_{\xi \eta}^{2} v_{2}}
\end{aligned}
$$

The motion is periodic with the periods $t_{1}=2 \pi / v_{1}$ and $t_{2}=2 \pi / v_{2}$ when the initial conditions are close to the triangular equilibrium point. Since $\mu$ is small the solution for the frequencies is given as

$$
\begin{aligned}
& v_{1,2}=-\lambda^{2}=\frac{1}{2}\left[\left(4-\frac{3}{\sqrt{\left(1-e^{2}\right)}}-\frac{8 A_{2} K_{2}}{\sqrt{\left(1-e^{2}\right)}}-\frac{3 A_{2} \mu}{\sqrt{\left(1-e^{2}\right)}}-\frac{2 A_{2} \varepsilon}{3 \sqrt{\left(1-e^{2}\right)}}\right) \pm\left(\left(4-\frac{3}{\sqrt{\left(1-e^{2}\right)}}-\frac{8 A_{2} K_{2}}{\sqrt{\left(1-e^{2}\right)}}-\frac{3 A_{2} \mu}{\sqrt{\left(1-e^{2}\right)}}-\frac{2 A_{2} \varepsilon}{3 \sqrt{\left(1-e^{2}\right)}}\right)^{2}-\right.\right. \\
& \left.\left.\left(\frac{27}{\left(1-e^{2}\right)} \mu(1-\mu)+\frac{6 \mu \varepsilon}{\left(1-e^{2}\right)}(1-\mu)+\frac{A_{2} \mu}{18\left(1-e^{2}\right)}\left(324+2052 K_{2}+2079 e^{2} K_{2}\right)\right)\right)^{0.5}\right] .
\end{aligned}
$$

Using the power series, the above frequencies can be determined. The conditions of stability in ER3BP is well satisfied by the Sun-Jupiter system, where $\mu=0.000953356$ and $e=0.04838624$. Applying these values in Eq. (54), the short- non-dimensional period of oscillation $t_{1}=6.32$ and the long- non-dimensional period of oscillation $t_{2}=76.032$ are determined. The orbital period of the Jupiter is $P=$ 11.862 years. Then the dimensional periods can be obtained as $T_{1}=P / v_{1}=11.932$ years and $T_{2}=P / v_{2}=143.54$ years as shown in Fig 9. The same periods computed for the circular problem provides $T_{1}=11.90$ years and $T_{2}=147.4$ years (Szebehely, 1967). So, it is clear that the long-periodic orbit is affected more by the influence of the eccentricity of the planet's orbit as it is shown by the reduction of its period when compared with the classical circular problem. 


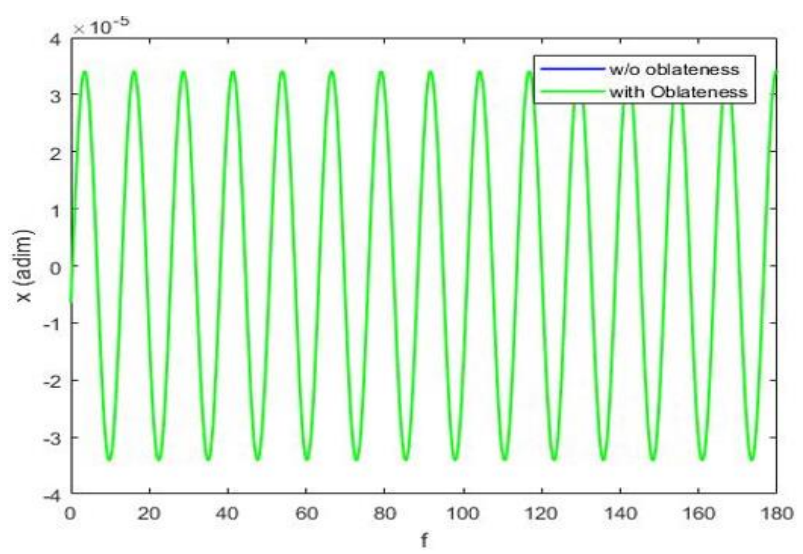

Fig. 9: Period of Oscillation for Sun-Jupiter System.

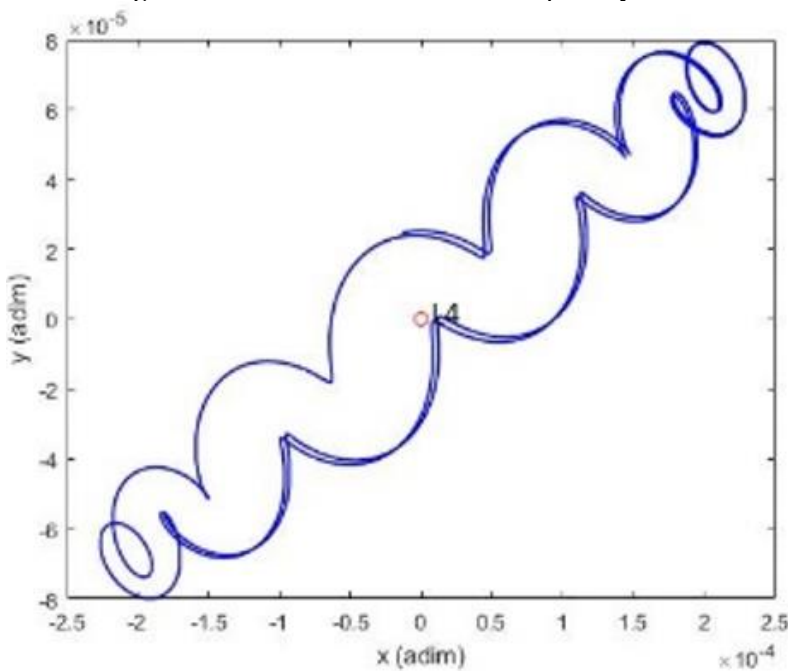

Fig. 10: Tadpole Orbit Around $\mathrm{L}_{4}$ Point for Sun-Jupiter in A Rotating-Pulsating Coordinate System.

A linear approximation of tadpole orbit provided by the combination of long- and short- period motions around $\boldsymbol{L}_{\mathbf{4}}$ from Eq. (49) in the Sun-Jupiter system for the 20 complete revolutions is shown in Fig (10).

\subsection{Motion around L4/ L5 in the case of perturbations}

When perturbations due to oblateness and radiation pressure is considered potential function is of the form (Ruth and Sharma [10])

$\Omega=\frac{1}{2}\left(\xi^{2}+\eta^{2}\right)+\frac{1}{n^{2}}\left\{\frac{(1-\mu) q}{r_{1}}+\frac{\mu}{r_{2}}+\frac{\mu A_{2}}{2 r_{2}^{3}}\right\}$,

where $\tilde{n}^{2}=1+2 K_{2} A_{2}\left[1+\frac{7}{4} e^{2}\right]$ is the mean motion, $A_{2}$ is the oblateness of smaller primary. It is computed from the general formula

$A_{2}=\frac{R_{e}^{2}-R_{p}^{2}}{5 R^{2}}$

where $R_{e}$ is the equatorial radius, $R_{p}$ is the polar radius and $R$ is the distance between the primaries.

Triangular position values are

$\xi=\mu-\frac{1}{2}+\frac{A_{2}}{2}-\frac{\varepsilon}{3}+\frac{4}{9} A_{2} K_{2} \varepsilon$

$\eta= \pm \frac{\sqrt{3}}{2}\left[1+\frac{2}{9} \varepsilon+\frac{A_{2}}{3}-\frac{8}{9} A_{2} K_{2}-\frac{8}{27} A_{2} K_{2} \varepsilon\right]$

Differentiating Eq. (55), we get

$\Omega_{\xi \xi}=1-\frac{1}{\tilde{n}^{2}}\left(\frac{3 A 2 \mu}{2 r 2^{5}}+\frac{\mu}{r 2^{3}}-\frac{15 A 2(1+\xi-\mu)^{2} \mu}{2 r 2^{7}}-\frac{3(1+\xi-\mu)^{2} \mu}{r 2^{5}}+\frac{(1-\mu)(1-\varepsilon)}{r 1^{3}}-\frac{3(1-\mu)(\xi-\mu)^{2}(1-\varepsilon)}{r 1^{5}}\right)$,

$\Omega_{\eta \eta}=1+\frac{\eta^{2}}{\tilde{n}^{2}}\left\{\frac{3(1-\mu)(1-\varepsilon)}{r 1^{5}}+\frac{3 \mu}{r 2^{5}}+\frac{15 A 2 \mu}{2 r 2^{7}}\right\}-\frac{1}{\tilde{n}^{2}}\left\{\frac{(1-\mu)(1-\varepsilon)}{r 1^{3}}+\frac{\mu}{r 2^{3}}+\frac{3 A 2 \mu}{2 r 2^{5}}\right\}$,

$\Omega_{\xi \eta}=\frac{\eta}{\tilde{n}^{2}}\left\{\frac{3(1-\mu)(1-\varepsilon)(\xi-\mu)}{r 1^{5}}+\frac{3 \mu(1+\xi-\mu)}{r 2^{5}}+\frac{15 A 2(1+\xi-\mu) \mu}{2 r 2^{7}}\right\}$

Frequencies corresponding to the characteristic equation with the above value is given by Eq. (54). 
Consider Sun- Saturn system where $\mu=0.000285635$, $\mathrm{e}=0.05386179, \varepsilon=A_{2}=0$. Periods are $t_{1}=6.303$ and $t_{2}=142.4556$. Orbital period of Saturn is $\mathrm{P}=29.4571$ years. Hence the dimensional periods are $T_{1}=29.552$ years and $T_{2}=667.96$ years. Tadpole orbits developed around $L_{4}$ is shown in Fig 11 with 30 revolutions. The radiation and oblateness coefficients are $\varepsilon=0.0004, A_{2}=6.65 \times 10^{-11}$, respectively. With the effect of oblateness and radiation pressure, non- dimensional periods are $t_{1}=6.3031$ and $t_{2}=142.4370$ from Eq. (54). Hence the dimensional periods can be calculated as $T_{1}=29.552$ years and $T_{2}=667.96$ years. The orbit with the influence of oblateness and radiation pressure for 30 revolutions is shown in Fig 12.

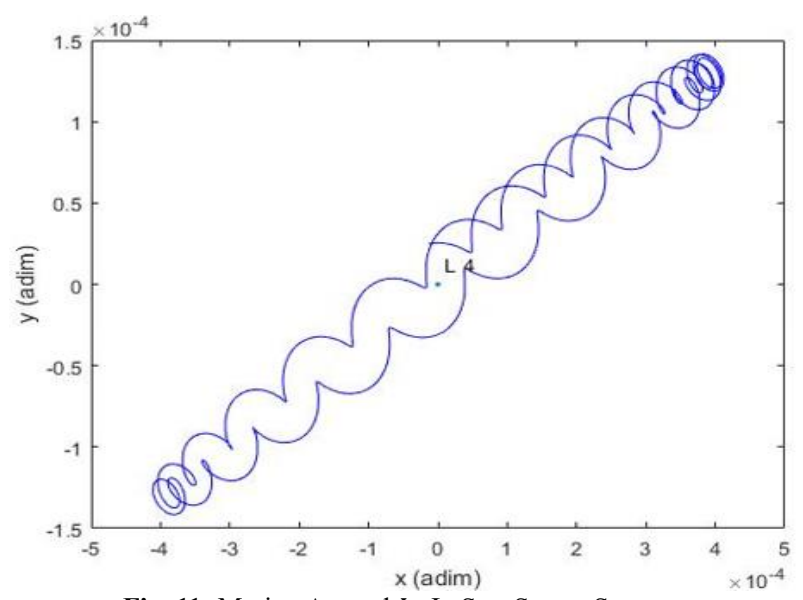

Fig. 11: Motion Around $L_{4}$ In Sun-Saturn System.

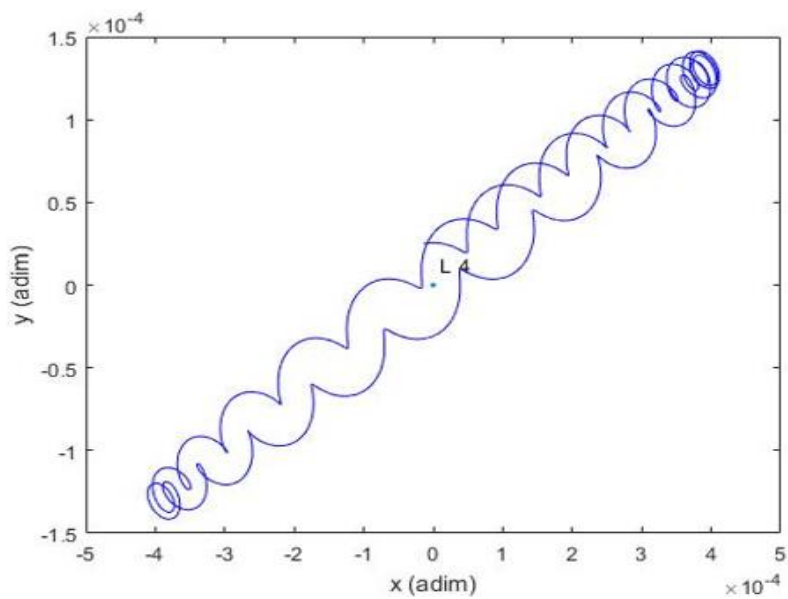

Fig. 12: Motion Around $L_{4}$ in Sun-Saturn System for when $\mathrm{E}=A_{2}=030$ Revolution when $\mathrm{E}=0.004, A_{2}=6.65 \times 10^{-11}$

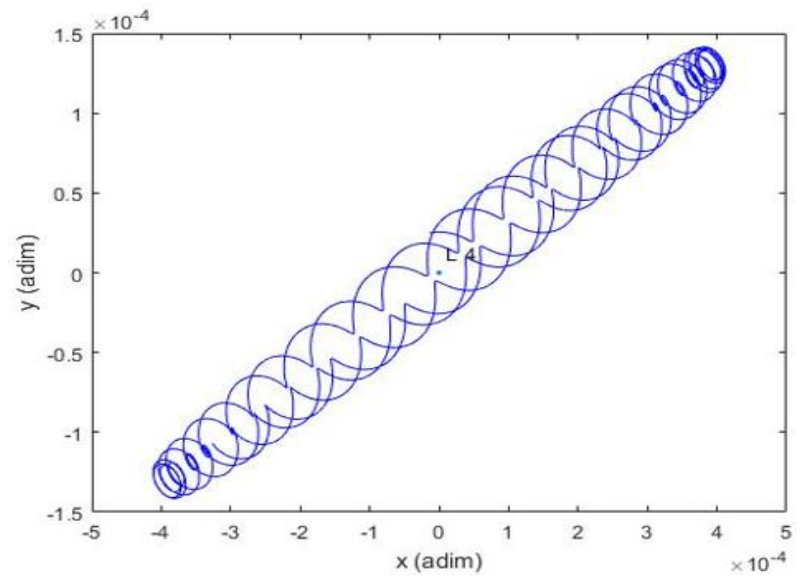

Fig. 13: Motion Around $L_{4}$ in Sun-Saturn System for. 


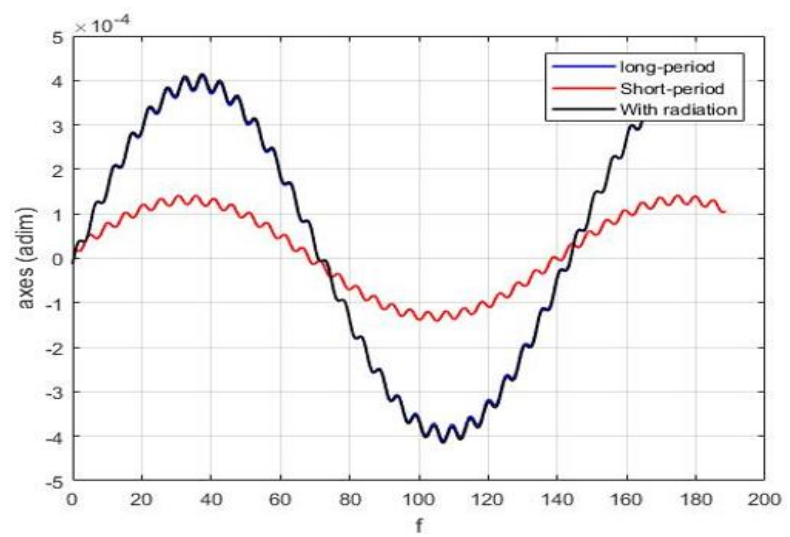

Fig. 14: $\mathrm{F} \epsilon[0,60 \pi]$ with $\mathrm{X}-\mathrm{Y}$ Axes for Combined Long 60 Revolution When $\mathrm{E}=0.004, A_{2}=6.65 \times 10^{-11}$ And Short Periodic Orbits in the Case of SunSaturn System with and Without Radiation.

The epicyclic motion (small ellipse) and motion of the epicenter (large ellipse) for Sun-Saturn system is shown in Fig 14 which forms a tadpole orbit. The particle's path around the equilibrium is a combination of the epicyclic motion and the motion of the epicenter.

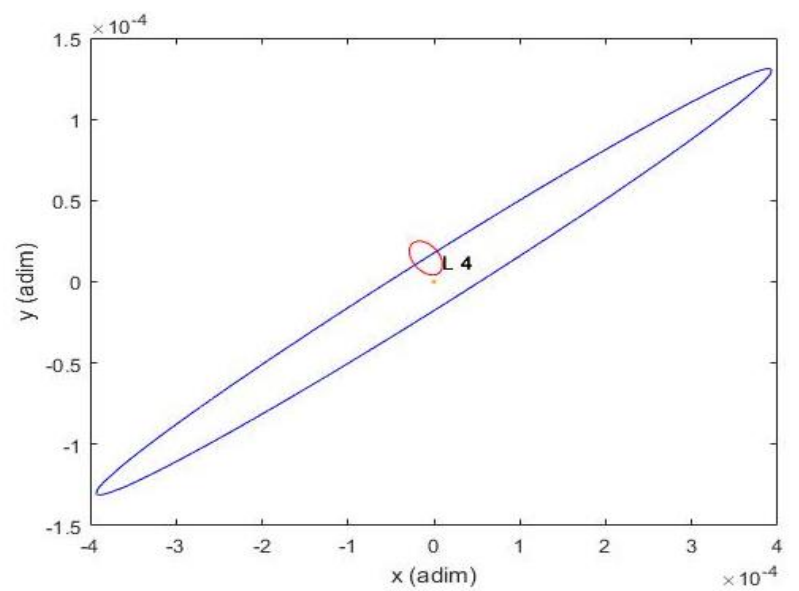

Fig. 15: The Long and Short Periodic Motion Around $L_{4}$ in Sun-Saturn System.

\section{Conclusion}

The locations of $\mathrm{L}_{1}, \mathrm{~L}_{2}, \mathrm{~L}_{3}$ and their stability are studied. The critical value of mass parameter $\mu_{c}$ is obtained. It decreases with the increase in radiation pressure and oblateness. This critical value is used to determine the size of the region of stability. The transition curve separates the stable region from the unstable region. The stability of the triangular points decreases with the increase in radiation pressure and oblateness. The zone of stability shrinks when the radiation pressure and oblateness increase. The tadpole orbits for Sun-Jupiter system with distinguished long- and short- periodic orbits for 30 revolutions are plotted. The periods of the short- and long- periodic orbits show a change from the relative circular problem for the same model. While the short period does not show much deviation, it indicates significant influence of the Jupiter's eccentricity in the librational motion of tadpole orbit.

\section{References}

[1] Danby. (1964). "Stability of the Triangular Points in the Elliptic Restricted Problem", The Astronomical Journal, 69, 165-172. https://doi.org/10.1086/109254.

[2] S.K. Sahoo and B.Ishwar (2000). "Stability of collinear equilibrium points in the generalized photogravitational elliptic restricted three-body problem". Astronomical Society of India, 28, 579-586.

[3] Jagadish Singh, Aishetu Umar (2012). "On out of plane equilibrium points in the ER3BP with radiating and oblate primaries". Astrophys, Space Science, 344, 13-19. https://doi.org/10.1007/s10509-012-1292-2.

[4] Szebehely, V. (1967). "Theory of Orbits, The Restricted Problem of Three bodies", Academic Press, New York. https://doi.org/10.1016/B978-0-12395732-0.50007-6.

[5] Broucke, R. A. (1969). "Periodic orbits in the Elliptic Restricted Three-Body Problem," JPL, California Institute of Technology, Pasadena, California, 32-1360. https://doi.org/10.2514/3.5267.

[6] Subba Rao, P. V. and Sharma, R. K. (1975). "A note on the Stability of the Triangular Points of the Equilibrium in the Restricted Three-body Problem". Astron and Astrophys. 43, 381-383.

[7] Raheem, A. and Singh, J. (2006). "Combined effects of perturbations, radiation, and oblateness on the stability of equilibrium points in the restricted three-body problem". The Astronomical Journal, 131, 1880-1885. https://doi.org/10.1086/499300.

[8] Isravel, H. and Sharma, R. K. (2017). "Effect of Oblateness on Transition Curves in Elliptic Restricted Three-body Problem". First International Conference on Recent Advances in Aerospace Engineering (ICRAAE). https://doi.org/10.1109/ICRAAE.2017.8297237.

[9] Grebenikov, E. A. (1964). "On the stability of the Lagrangian triangle solutions of the Restricted Elliptic Three-Body Problem", Soviet AstronomyAJ, 8, 451-459.

[10] Y. Sharon Ruth, Y. S. and Sharma, R. K. (2018). "Periodic orbits in the photogravitational Elliptical Restricted Three-Body Problem". Advances in Astrophysics, 3, 154-170.

[11] Murray, C. D. and S. F. Dermott, S. F. (1999). "Solar System Dynamics", Cambridge University Press, Cambridge, UK.

[12] Anderlecht, A. G. (2016), "Tadpole orbits in the L4/L5 region: Construction and links to other families of periodic orbits", MS Thesis, Purdue University, West Lafayette, Indiana, USA. 
[13] Sharma, R. K., Sellamuthu, H., Isravel, H. "Effect of oblateness on the locations and linear stability of collinear points in elliptic restricted threebody problem", communicated to Planetary and Space Science, August 2018.

[14] Bennett, A. (1965). "Analytical Determination of Characteristic Exponents," AIAA/ION Astrodynamics Specialist Conference, Monterey, California, No. 65-685. https://doi.org/10.2514/6.1965-685. 